Corresponding author:

Jörg Schacht

Mailing address:

Max-Planck-Institut für Plasmaphysik,

Teilinstitut Greifswald, Wendelsteinstr. 1,

D-17491 Greifswald,

Tel (+49) 3834 882761, Fax (+49) 3834882709 ,

E-Mail: Joerg.Schacht@ipp.mpg.de 


\title{
Overview and status of the control system of
}

\section{WENDELSTEIN 7-X}

Jörg Schacht, Heike Laqua, Marc Lewerentz, Ina Müller, Steffen Pingel, Anett Spring, Andreas Wölk

Max-Planck-Institut für Plasmaphysik, EURATOM Association,

Teilinstitut Greifswald, Wendelsteinstr. 1, D-17491 Greifswald

\begin{abstract}
The new quality of the superconducting fusion device WENDELSTEIN 7-X (W7-X) is its capability for long-pulse operation. Discharge operations can be up to 30 minutes with full heating power. Considering the steady state operation capability the W7-X system control will be important for future long-term fusion experiments. A wide spectrum of requirements has to be considered during its design and realization. The experimental nature of the W7-X project requires high flexibility for machine operation and automatic program controlled operation.
\end{abstract}

Main subject of this article is the description of a local control component as a part of the system control of W7-X. After a short overview about the structure of the system control a generic model of the local control components is described. This model permits a standardized procedure to integrate a component into the control system. The 
standard interfaces of the local components are described. Currently, some of the technical components of W7-X are already delivered, the others being in the phase of procurement.

Keywords: WENDELSTEIN 7-X; device control; operational management; segment control; plasma discharge control; safety system;

\section{Introduction}

The fusion experiment W7-X is the last large superconducting device in Europe before the start of ITER. The new quality of W7-X is its capability for long-pulse operation. The duration of a plasma discharge can be up to 30 minutes at full heating power. Considering the steady state operation capability the W7-X control system will be important for future long-term fusion experiments. A wide spectrum of requirements has to be considered during design and realization of the control system control. The experimental nature of W7-X requires high flexibility for machine operation and automatic program controlled operation. During the planned life cycle of about two decades the scientific programs will be permanently adapted to new operations. New components will be included into the control system and established systems have to be adapted with respect to technological progress. W7-X is a complex technical system consisting of a large number of subcomponents for technical, scientific, and infrastructure purposes [1]. The control system is hierarchically structured with the central control system at the top. Local components, e.g. power supplies, heating systems, and diagnostics, have their own control systems. For commissioning and tests local systems will be run autonomously. In preparation for experiment operation all essential components will be subordinated to the central control system. During 
experiments the central control system coordinates the activity of these components. A special segment control system is responsible for processing predefined programs.

It is an essential feature of the W7-X control system that technical processes are controlled and supervised by software. Therefore a highly developed, reliable and standard control technology and infrastructure must be used. The computer based W7$\mathrm{X}$ control system allows for a high flexibility of machine operation which is particularly required due to the experimental nature of $\mathrm{W} 7-\mathrm{X}$ operation and the long operating period. As far as possible, components of the machine are designed in respect of the steady state operation. The W7-X long pulse operation permits to divide the discharge into individual sections aiming at a number of experiments with different physical purposes during a plasma discharge. One section of the plasma discharge is called a segment. Each segment defines completely the behaviour of the components involved in the experiment at the time of the validity of the segment. Thus the planned plasma discharge consists of a chain of predefined segments to be carried out in real time.

In principle W7-X plasma operation can be divided into three types: Short discharges at variable interval can be used for commissioning of the device and during the initial phase of operation, as is usual in most existing fusion experiments (type A). The discharge phases have to be extended in subsequent operation to stationary long-time discharges (type B). A further possibility for structuring experimental operation is to subdivide a long-time discharge into short phases with different characteristics (type C). The definition of the desired characteristics for the phases of a discharge includes all the technical and scientific components involved in the execution of the experiment. A sequence of single segments can be summarized as a segment chain in order to simplify editing experiment programs, and a reuse of sections of proven experiments. The 
division of an extended discharge phase into segments reduces the time required for carrying out, for example parameter and diagnostic scans, and allows for short tests of new settings without affecting the main programme defined for the discharge phase.

\section{Control System overview}

All diagnostic components, the heating systems (ECRH, NBI, ICRH), power supplies for the magnetic coils are examples of locally controlled components. These components can be structured into two main groups: technical components (e.g. power supplies for the magnetic coils, heating systems, cryogenic system, and machine instrumentation) and scientific systems (all diagnostic systems). A subpart of the technical components are the auxiliary components. Examples for the auxiliary components are e.g. the gas supply system, the cooling system, all access control systems, fire alarm systems, and others. Both the technical components and the scientific components are only subtypes of a standard local control component. They are used for both device preparation and processing the experiment programs.

The control system is the basis for realization of the experiment programs of the physicists (see Fig. 1). It consists of the subcomponents central safety system, operational management, the slow sequence control, segment sequence control, monitoring, and visualization. The tasks of these central control components are described in [1] [2]. The control systems of the components are individually designed for each technical component. They have a few features in common which are necessary for coordinated operation. The Trigger Time Event system (TTE system) and the database serve all the parts of the control system at any time [4]. Four networks fulfill all demands concerning network communication on different levels. The TTE 
network is a unidirectional optical fiber network with a simple proprietary protocol. It is used for the distribution of the precise system time and for broadcasting event messages with real time requirements in the order of $10 \mu \mathrm{s}$. The standard network means the standard LAN running TCP/IP protocol and it is used for transmission of all information with no or minor real time requirements. The real time network is technologically similar but physically separated from the standard network. It is a simply structured switched Ethernet. Data packets are transmitted with the UDP protocol or with a very simple layer 2 protocol. Layer 2 switching, simple protocols, short data packets and low loading permit applications with real time requirements in the order of $100 \mu \mathrm{s}$. This network will be used for the distribution of event messages and of measured values. Multicast is commonly used.

The safety system is an independently working system and consists of local units responsible for the safety of each component and a central unit ensuring the safety of the whole W7-X system. Interactions with other components and with the environment, mainly persons at the experiment, are handled by the central safety system. It provides interlocks and controls human access to the device on the basis of six operational states (switched off, stand by, experiment pause, experiment operation, partially protected, and emergency stop). The interlocks and all safety related signals and messages are distributed via a safety bus and a safety signal network. A safety analysis on the base of the European Norm EN 1050: Safety of machines is the basis for the development of the safety system.

Entries in the configuration and segment program data base are organized in data structures of projects and components. All components of a control system represent a project, e.g. the W7-X is a project including more than 40 components for the first 
operation phase. This differentiation permits to configure and to control other laboratory systems and experimental devices with the same methods and tools if they are using the W7-X control system architecture. There are two possible modes for the authorization to control a group: the subordinated and the autonomous mode (confer Fig.2). The group control systems of a project are able to run in autonomous mode for commissioning and tests. If the autonomous mode is active a group can be controlled by its local operational management. Also segment control can be used for tests, calibration, or other complex operations in the autonomous mode of a group, independent of the project's central control system. In this operational mode the component segment control processes the predefined group segment programs.

If the central control system coordinates the control systems of the components during experiments or other phases requiring operation of the device all necessary local components are sub-ordinate to the central control system. The central operator observes and controls the subordinated components by means of the central operational management. After all necessary local components are prepared the processing of project segment programs can be started. An interactive session leader program Xcontrol [3] allows the responsible operator to choose and chain predefined segment programs, to start or stop a segment program chain as a discharge. The progress of the discharge is visualized by a sequence monitor.

\section{Model of a local control component}

Each technical and diagnostic component of the W7-X project can be described by a unified component model. This model defines the internal parts of a component and the communication between the component's substructures and the central control system. 
In such a way, this component model permits a standardized procedure to integrate a local control component into the control system of W7-X.

In general there is no basic difference between the control systems of technical and scientific components. However, the implementation will be strongly different because of different requirements of the different components.

A W7-X control component has a distributed control system which consists of modules with different functionality. Equivalent to the central control system the control system of a local component is structured into a safety system, an operational management, and a segment control system. The local safety system supervises the operation of the component and activates the "safe state" of a component in case of serious faults. The safety system of the component closely interacts with the central safety system. Via a safety interface, realized in safety technique, signals will be transmitted for enabling of dangerous components activities, and for activating the safe state of the component. All components of the safety interface and of the central safety system fulfill the requirements of the safety integrated level: SIL 3.

The so called local operation management (IOPM) is responsible for all slow control operation of a component. The operator can change the operational state of his component by means of IOPM. Each operational state is defined for performing dedicated activities, for example switching On/Off, configuring the devices of the component in a preparation mode, calling predefined functions or enables the component for the automatic processing of segment programs in autonomous or subordinated mode. Switching between the autonomous and the subordinated mode is implemented in the IOPM, too.

Some technical processes, e.g. baking and conditioning of the vacuum vessel, are 
started by the central slow sequence control but the necessary processes are running under control of the IOPM. Standard industrial products are used for the realization of lOPM. For slow control processes the Siemens SIMATIC S7 PLCs (PLC: Programmable Logic Controller) is used. For visualization, another important task of the IOPM, the Siemens WinCC program in a client-server configuration has been chosen.

The elements of a generic local control system are shown in Fig. 3. At the bottom of this structure - the field level - are located the sensors and the actuators. The control level above the field level realizes all necessary functions for control and data processing. Slow control processes are managed by PLCs. In cases with more than one PLC a master / slave configuration is used. Control processes with real time requirements are done on standard PCs in 19', casing running the VxWorks operating systems (FCS: fast control station). Data acquisition with a high level of data amounts is the task for Data Acquisition and Control stations (DAQ station). The DAQ stations are equipped with a normal operating system such as Windows XP or Linux. The programs for the DAQ stations are written in JAVA to be independent of the operating system. Both, the FCS and the DAQ's are responsible to control the behaviour of the component during experiments by segment control system. The special function of a "speaker" is necessary, if the component is equipped with more than one control station (CS). The speaker is the interface between the component's segment controllers and operational management as well as to the central segment control system. Mainly it summarizes all status information about the control station's operational state and of the status of segment processing and sends this information periodically to all defined receivers.

The data management level is the highest level of the component model. In this level all 
functions for visualization, controlling and supervising of the component are realized. By using of a GUI (graphical user interface) the local operator can control and observe the component.

The standardization of the structure and tasks of a local control component allows a standardization of its internal and external communication paths, too. The messages consist of a standard part and an individual part defined corresponding to the individual character of the component. The data flows of the system control are divided into three main lines, as shown in Fig.4. The safety system is using a special field bus (Profi Safe) and a dedicated signal network. The communication between the components of the slow control system consisting of central slow sequence control and operational management is based on normal Ethernet. The fast control system sends messages via Ethernet. A fast data network (Real Time Data Bus) is used for a periodic data exchange with demanding real time conditions. The connection of the control nodes (PLC's and Control Stations) with theirs sensors and actors of the field level is very different and depends on the given interface types (e.g. signal networks, field busses) of the involved interface components. A data exchange between the control nodes is realized via Ethernet.

One example for a standard communication is the message of a local control component status. The status of a component must be built periodically every second and after state changes. All control nodes of a component calculates its own operational status. A slave PLC sends its status message to the master PLC and a control station sends it to the Speaker CS. The Speaker has the task to build a status of all CS of the component and send it to the master PLC. The master PLC is now able to build a complex operational status of the whole component. The content of the component status message consist 
among other things of the status of communication, the operational state (OFF, STANDBY, PREPARE, ON, ...), information about the authority to control, warnings and errors states, readiness for segment control operations, set values, actual values, limit values. Receiver for the status messages of all project components is the central operational management (cOPM). The cOPM analyzes the status messages and visualized them. Furthermore all components' status information has to be summarized as a status message of the project. Using the multicast protocol this project status message is sent to the session leader program and to the central segment sequence controller for segment control purposes. Another example for a standard message is the segment switch command. This command is sent as multicast message by the central sequence control system to all segment controllers of project. This message contains the identification of the next segment program. After receiving this message all control stations must activate the new segment program immediately.

\section{Status of realization}

Currently, some of the technical components of W7-X are already delivered, the others being in the phase of procurement. Already finished components are used for testing their control systems and interfaces. The central control system for W7-X is under construction. Prototypes of all subsystems of the central control are tested. The segment control system can be used for tests in a project or group configuration.

The next important step will be an integrated test of the W7-X system control concepts. The WEGA device situated in IPP branch Greifswald is a small stellarator experiment. In a planned reconstruction phase of about 2 years WEGA shall be equipped with a W7X like control system and can be tested under real plasma operation conditions. 


\section{Acknowledgements}

The authors would like to thank the data acquisition group at IPP for prototype testing, recommendation and lots of helpful discussions.

\section{References}

[1] J. Schacht, H. Niedermeyer, H. Laqua, A. Spring, I. Müller, St. Pingel and A. Wölk: Tasks and structure of the WENDELSTEIN7-X control system, Fusion Engineering and Design, to be published, Corrected Proof available online at www.sciencedirect.com 22 May 2006.

[2] H. Laqua, H. Niedermeyer, J. Schacht, A. Spring: Real-time Software for the Fusion Experiment WENDELSTEIN 7-X, Fusion Engineering and Design, to be published, Corrected Proof available online at www.sciencedirect.com 5 June 2006.

[3] A. Spring, H. Laqua, J. Schacht: User Control Interface for W7-X Plasma Operation, Fusion Engineering and Design - this issue.

[4] J. Schacht, H. Laqua, and H. Niedermeyer, Synchronization of processes in a distributed real time system exemplified by the control system of the fusion experiment WENDELSTEIN 7-X, Proc. 14th IEEE-NPSS Real Time Conference, Stockholm, Sweden, 2005. 


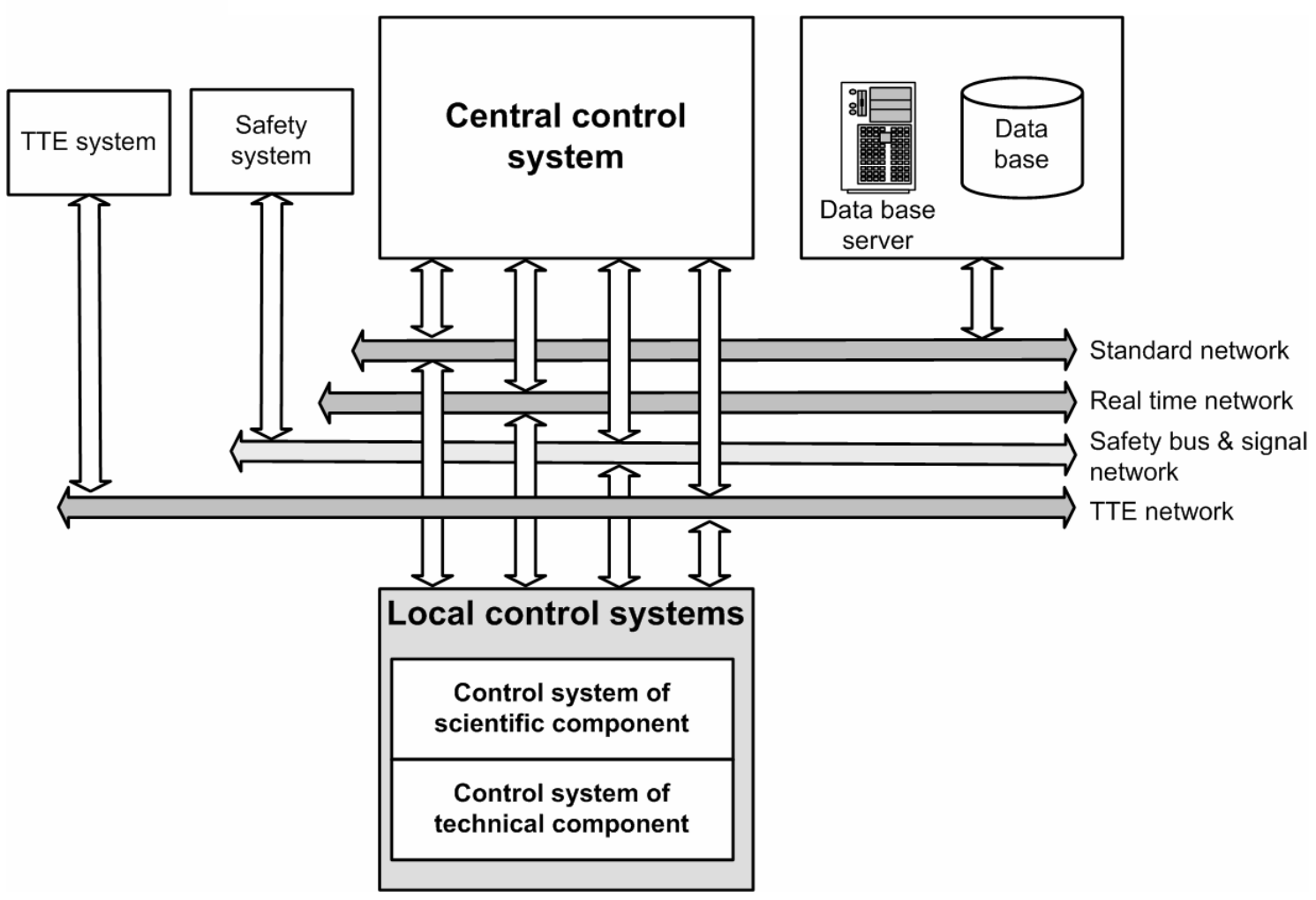

Fig. 1: Structure of the W7-X system control 


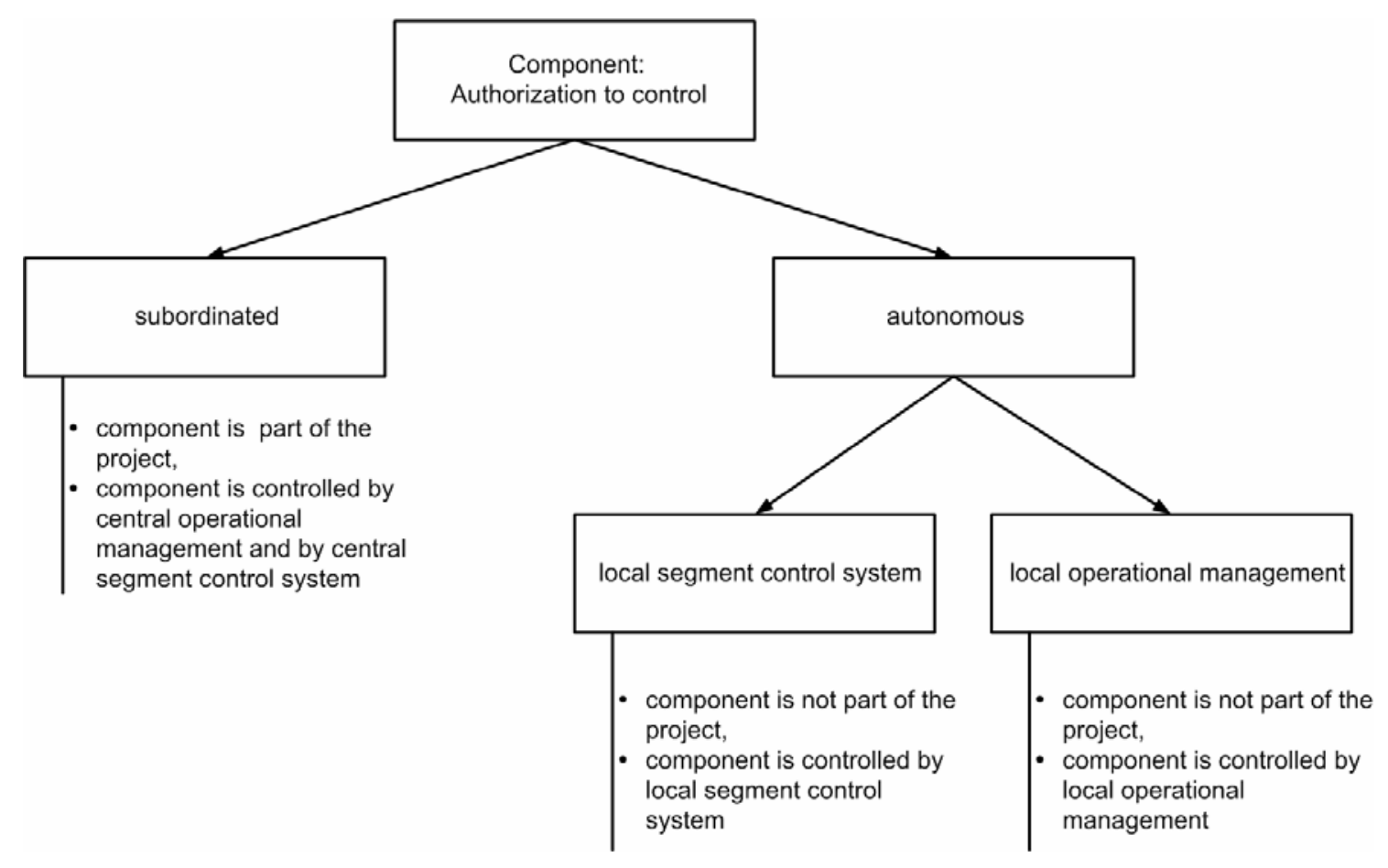

Fig. 2: Modes for authorization to control a control component 

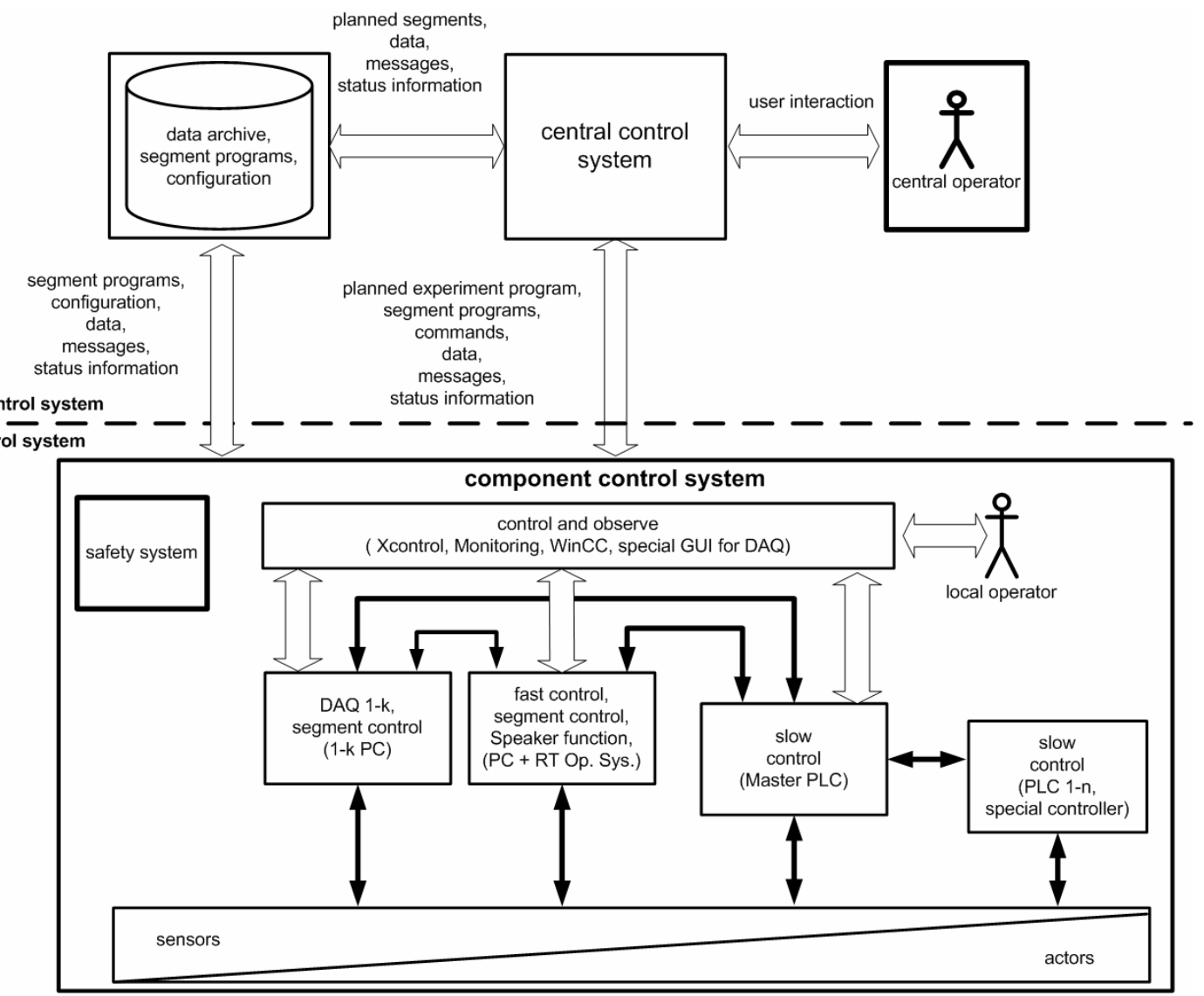

Fig. 3: Structure of a W7-X component control system 


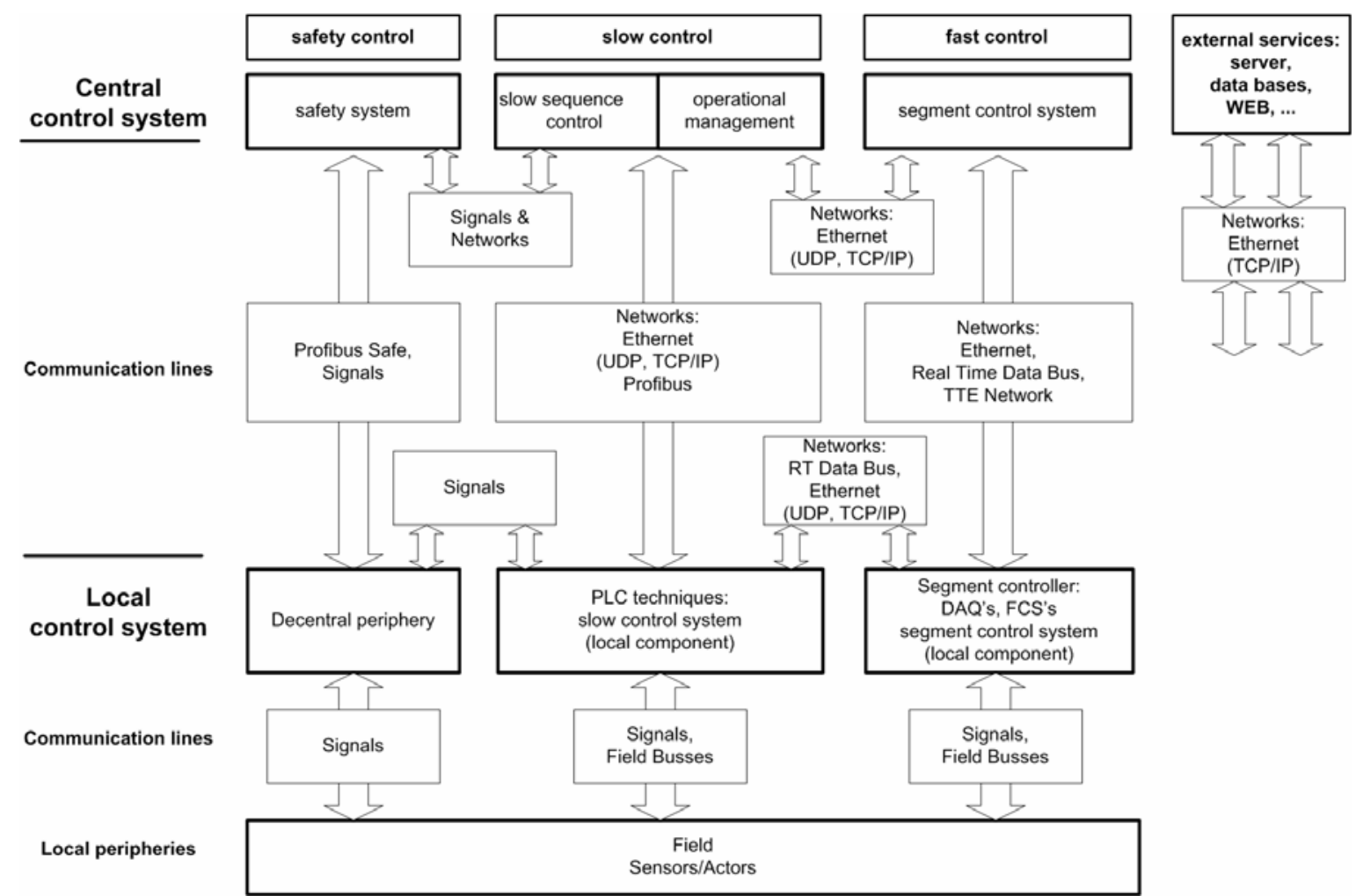

Fig. 4: Communication lines of system control 